\title{
Beginning \\ Samsung ARTIK
}

A Guide for Developers

Cliff Wootton

Apress $^{\oplus}$ 


\section{Beginning Samsung ARTIK}

\section{Cliff Wootton}

Crowborough, East Sussex, United Kingdom

ISBN-13 (pbk): 978-1-4842-1951-5

ISBN-13 (electronic): 978-1-4842-1952-2

DOI 10.1007/978-1-4842-1952-2

\section{Library of Congress Control Number: 2016940104 \\ Copyright (C) 2016 by Cliff Wootton}

This work is subject to copyright. All rights are reserved by the Publisher, whether the whole or part of the material is concerned, specifically the rights of translation, reprinting, reuse of illustrations, recitation, broadcasting, reproduction on microfilms or in any other physical way, and transmission or information storage and retrieval, electronic adaptation, computer software, or by similar or dissimilar methodology now known or hereafter developed. Exempted from this legal reservation are brief excerpts in connection with reviews or scholarly analysis or material supplied specifically for the purpose of being entered and executed on a computer system, for exclusive use by the purchaser of the work. Duplication of this publication or parts thereof is permitted only under the provisions of the Copyright Law of the Publisher's location, in its current version, and permission for use must always be obtained from Springer. Permissions for use may be obtained through RightsLink at the Copyright Clearance Center. Violations are liable to prosecution under the respective Copyright Law.

Trademarked names, logos, and images may appear in this book. Rather than use a trademark symbol with every occurrence of a trademarked name, logo, or image we use the names, logos, and images only in an editorial fashion and to the benefit of the trademark owner, with no intention of infringement of the trademark.

The use in this publication of trade names, trademarks, service marks, and similar terms, even if they are not identified as such, is not to be taken as an expression of opinion as to whether or not they are subject to proprietary rights.

While the advice and information in this book are believed to be true and accurate at the date of publication, neither the authors nor the editors nor the publisher can accept any legal responsibility for any errors or omissions that may be made. The publisher makes no warranty, express or implied, with respect to the material contained herein.

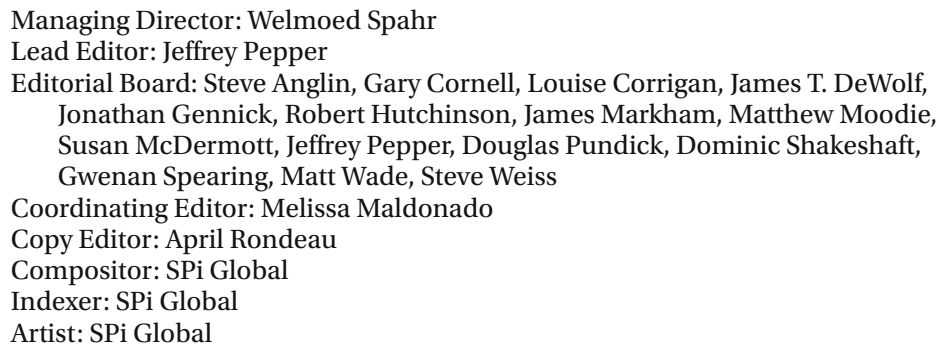

Distributed to the book trade worldwide by Springer Science+Business Media New York, 233 Spring Street, 6th Floor, New York, NY 10013. Phone 1-800-SPRINGER, fax (201) 348-4505, e-mail orders-ny@springer-sbm.com, or visit www.springer.com. Apress Media, LLC is a California LLC and the sole member (owner) is Springer Science + Business Media Finance Inc (SSBM Finance Inc). SSBM Finance Inc is a Delaware corporation.

For information on translations, please e-mail rights@apress.com, or visit wWw.apress.com.

Apress and friends of ED books may be purchased in bulk for academic, corporate, or promotional use. eBook versions and licenses are also available for most titles. For more information, reference our Special Bulk Sales-eBook Licensing web page at wWw. apress . com/bulk-sales.

Any source code or other supplementary materials referenced by the author in this text is available to readers at WWW. apress. com. For detailed information about how to locate your book's source code, go to to wWw. apress. com/source-code/.

Printed on acid-free paper 
To my longtime buddy, Pete Willis. 



\section{Contents at a Glance}

About the Author …...................................................................xxvii

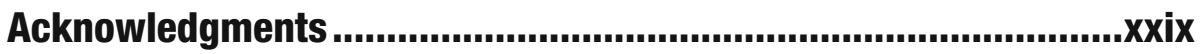

Trademarks......................................................................................xxxi

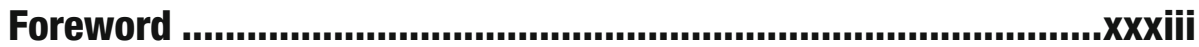

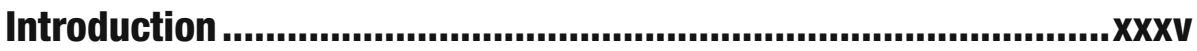

Chapter 1: Getting Started .........................................................

Chapter 2: Welcome to the Internet of Things .............................. 9

Chapter 3: Hello, ARTIK....................................................................15

Chapter 4: Understanding Security ........................................... 41

Chapter 5: Your Development Kit..................................................... 47

Chapter 6: Getting Your Hardware Together .................................. 59

Chapter 7: Setting Up a Terminal Emulator ...................................... 81

Chapter 8: Talking to Your ARTIK............................................. 99

Chapter 9: Network Configuration ......................................... 109

Chapter 10: Configuring and Upgrading ..................................... 143

Chapter 11: Programming Your ARTIK......................................... 159

Chapter 12: Using Eclipse IDE .............................................. 175

Chapter 13: Using Arduino IDE ............................................. 195

Chapter 14: Using the Command Line .................................. 219 
Chapter 15: Programming in C Language............................... 239

Chapter 16: Programming with Node.js ................................. 257

Chapter 17: Programming with Python ................................... 267

Chapter 18: Integrating with SAMI .......................................... 273

Chapter 19: Integrating with Temboo ..................................... 301

Chapter 20: Debugging Your Application................................... 325

Chapter 21: Deploying Your Application ..................................... 333

Chapter 22: Next Steps....................................................... 341

Index......................................................................................... 347 


\section{Contents}

About the Author …...................................................................xxvii

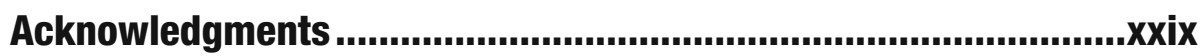

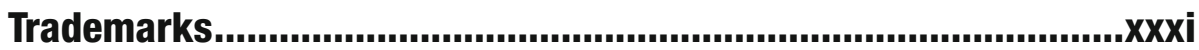

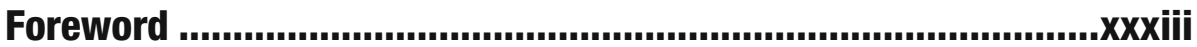

Introduction .................................................................................XXXV

Chapter 1: Getting Started ......................................................... 1

Introducing the ARTIK Family ........................................................... 1

Reinventing the Engineering Process................................................. 1

About This Book ……................................................................. 2

Finding Out About ARTIK.............................................................. 2

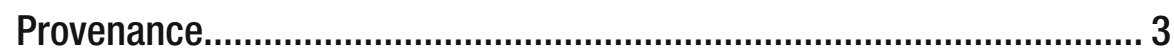

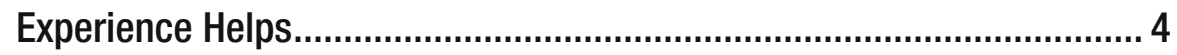

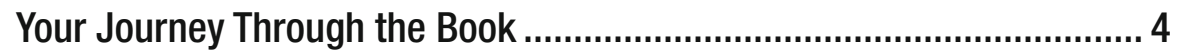

Do You Use Windows, Linux, or Mac OS X? ......................................... 4

Get Your Samsung Account ............................................................ 5

Buy an ARTIK Development System Now .......................................... 5

Would You Like to Know More? ......................................................... 5

What to Read Next........................................................................... 6

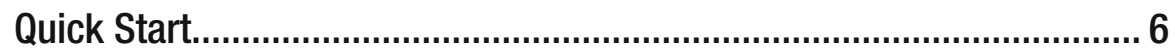


Chapter 2: Welcome to the Internet of Things ............................... 9

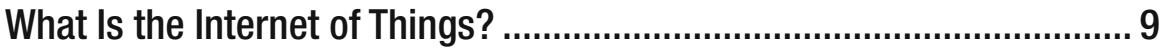

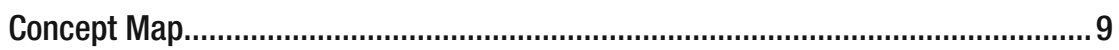

An Example You Can Build Today .......................................................................10

The Internet Is Changing—Rapidly ................................................... 10

Disruptive Changes......................................................................................11

Network Architecture and Design ......................................................................11

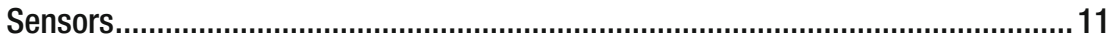

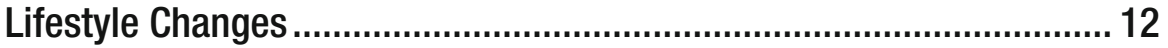

A Revolution in Medicine and the Care Community...........................................12

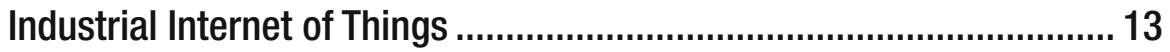

Revenue-generating Opportunities..................................................................13

Chapter 3: Hello, ARTIK............................................................ 15

What Is an ARTIK Module?.................................................................. 15

What Is an Ecosystem? ......................................................................... 15

The ARTIK Modules............................................................................... 16

Software Support ................................................................................ 17

The ARTIK Community .....................................................................................17

Security and Privacy Are Core Needs ...................................................... 18

The Connected Cloud Service ............................................................... 19

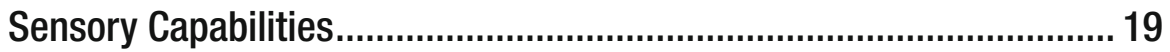

Communications Challenges ................................................................ 19

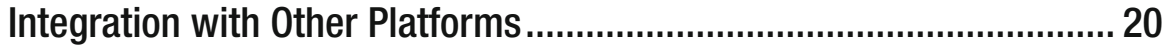

Benefits to Consumers .......................................................................... 21

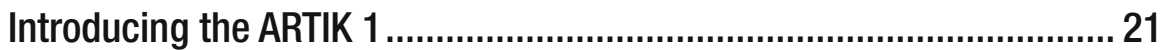

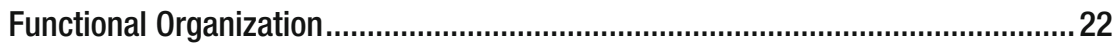

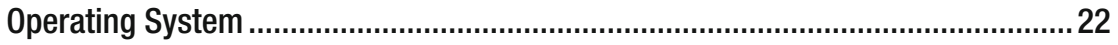




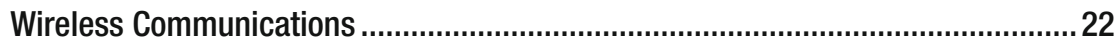

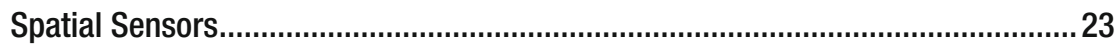

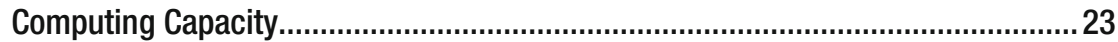

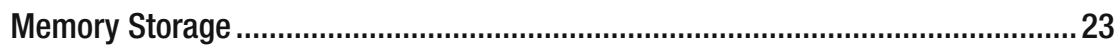

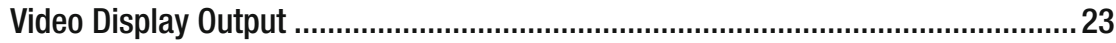

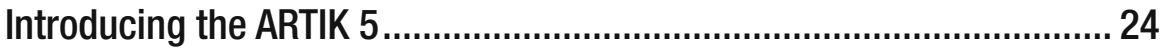

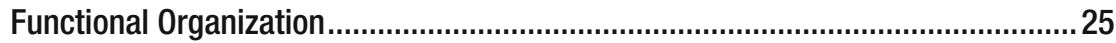

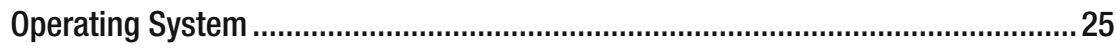

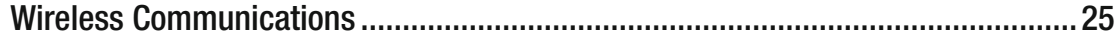

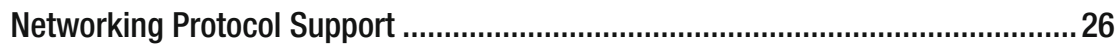

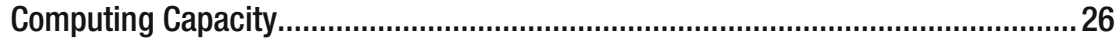

Graphics Processing Unit (GPU) ................................................................. 26

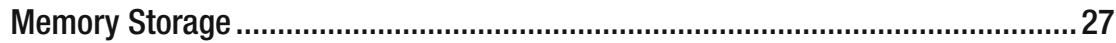

Hardware Video Codec Support..........................................................................2

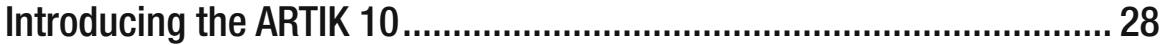

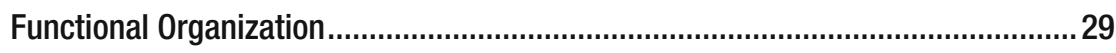

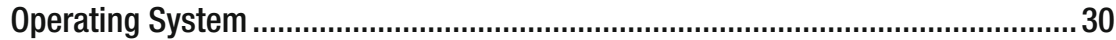

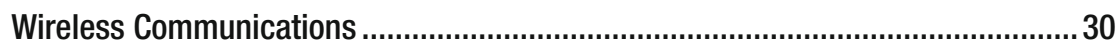

Networking Protocol Support ............................................................................... 31

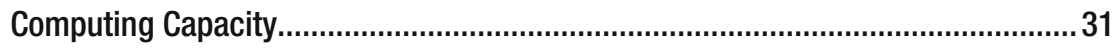

Graphics Processing Unit (GPU) ...................................................................... 31

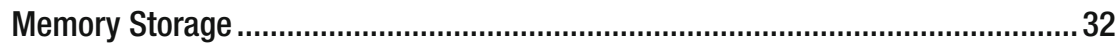

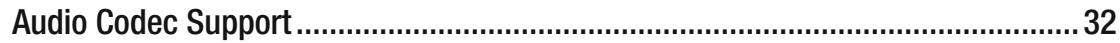

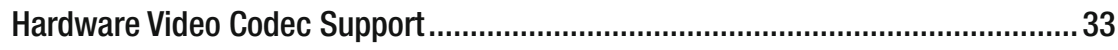

Comparing the ARTIK Modules ................................................................. 33

Power Management Integrated Circuit (PMIC) ………........................................... 33

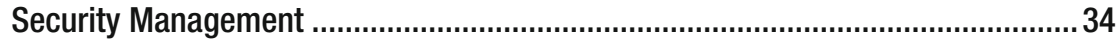

Support for Audio Coding................................................................................ 34 


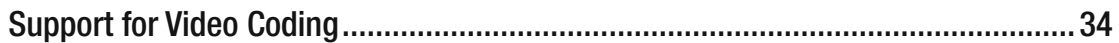

Physical Connections................................................................................... 35

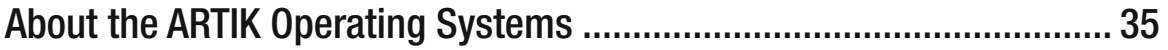

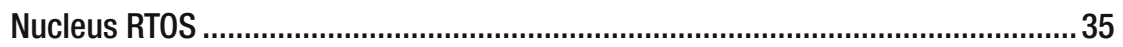

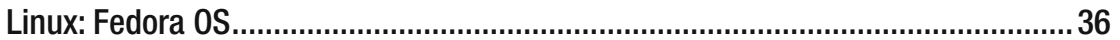

But What Is Yocto? ................................................................................... 37

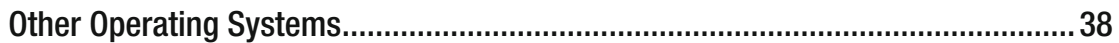

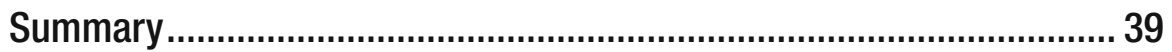

Chapter 4: Understanding Security ............................................ 41

Risk Factors and Dystopian Futures..................................................... 41

Security Ecosystem ............................................................................... 42

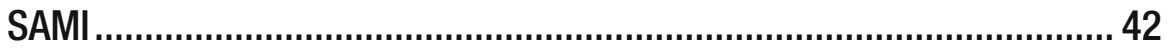

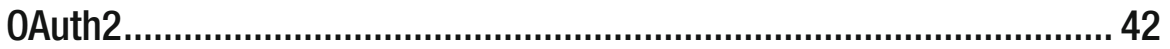

Cloud-based Services …........................................................................ 43

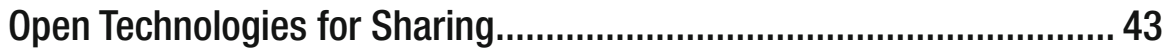

Keeping Your Data Safe and Secure................................................... 43

Secure Operating System................................................................. 43

Firmware Security ....................................................................... 44

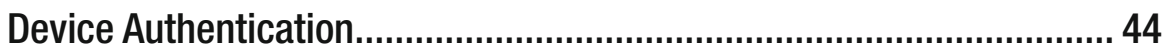

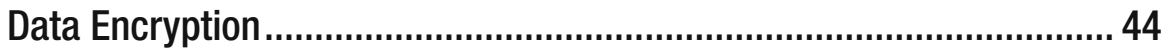

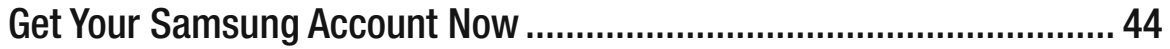

Embedded Secure Element ................................................................. 45

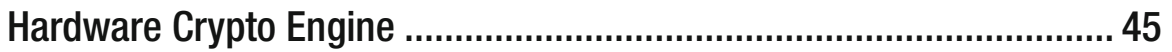

Segregated Trust Zone .................................................................. 46

Current Status ........................................................................... 46

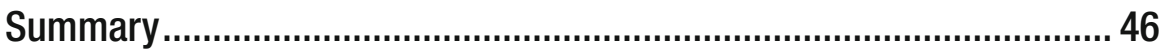


Chapter 5: Your Development Kit

The Developer Reference Board..

About the Developer Reference Boards............................................................... 47

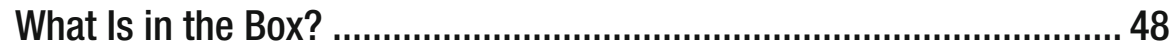

The Type 1 Developer Reference Board.............................................. 50

The Type 5 \& 10 Developer Reference Boards (Beta) ............................ 51

Early Production Models..................................................................... 53

Type 5 and 10 Developer Reference Board Connectors ....................... 53

Type 5 and 10 Developer Reference Board Switches........................... 55

Type 5 and 10 Developer Reference Board LED Indicators .................. 55

Type 5 and 10 Developer Reference Board Jumpers ........................... 56

Connecting External Devices.............................................................. 56

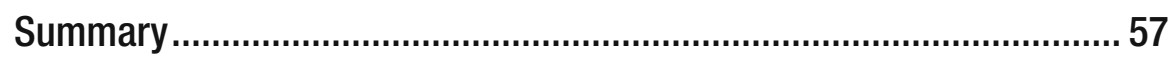

Chapter 6: Getting Your Hardware Together ................................. 59

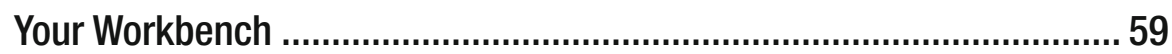

Setting Up a Hardware Workbench .................................................... 59

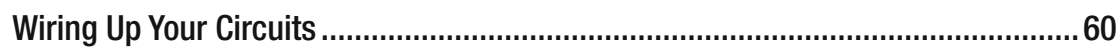

Test Equipment.......................................................................................... 61

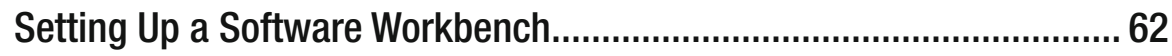

Risk Managing Your Software Development.........................................................63

Configuring the Developer Reference Board ....................................... 63

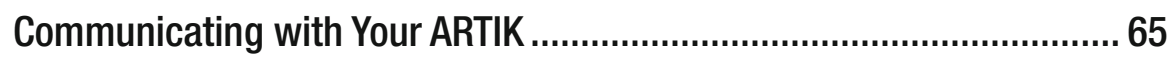

Connecting the ARTIK Development System ........................................66

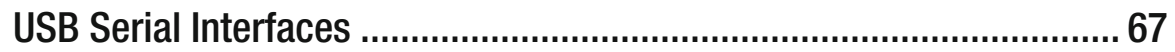

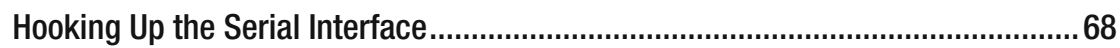

Setting Up a USB Serial Interface Driver on Windows ........................................... 68

Setting Up a USB Serial Interface Driver on Mac OS X .........................................69 
Setting Up a USB Serial Interface Driver on Ubuntu Linux......................................75

Setting Up a USB Serial Interface on Android Devices .........................................77

USB Vendor IDs..................................................................................... 78

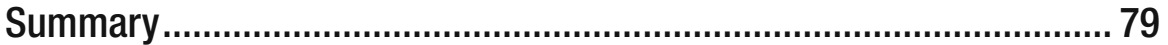

Chapter 7: Setting Up a Terminal Emulator ............................... 81

Serial Connections with a Terminal Emulator....................................... 81

Installing Your Terminal Emulator......................................................... 82

Adding a Terminal Emulator to Windows............................................. 82

Install PuTTY on Windows.............................................................................. 82

Connecting to the ARTIK Development System from PuTTY....................................84

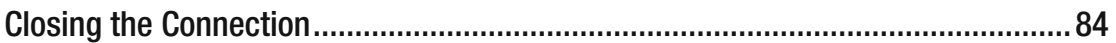

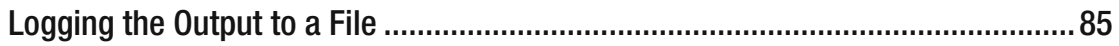

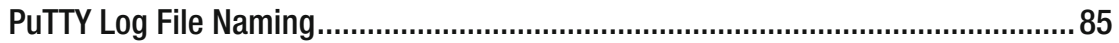

Using the Default Terminal Application on Mac OS X ........................... 86

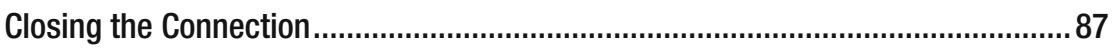

Recovering from a Bad Screen Exit..................................................................... 88

Other Useful Screen Commands.................................................................... 89

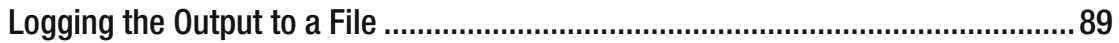

Log Capture Example 1 (Clipboard Cut and Paste) ...............................................90

Log Capture Example 2 (Screen Command Logging) ...........................................90

Log Capture Example 3 (Output Redirection)........................................................91

Log Capture Example 4 (Stream Duplexing) .........................................................91

Log Capture Example 5 (Script Command Logging) …….....................................92

Alternatives to the Mac OS X Terminal App .....................................................92

Using the Minicom Terminal Application on Linux.................................92

Installing Minicom with yum ...........................................................................93

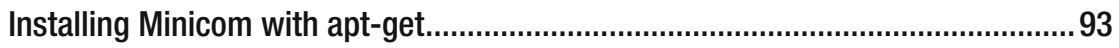

Building Minicom from the Source Code Files.......................................................93 
Configure Minicom to Talk to the ARTIK Developer Reference Board ......................94

Connecting to the ARTIK Development System from Minicom ..............................95

Closing the Connection................................................................................96

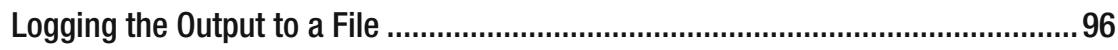

Pausing the Screen Output...............................................................................97

Using Minicom Inside Your ARTIK..................................................... 97

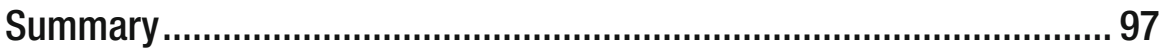

Chapter 8: Talking to Your ARTIK................................................... 99

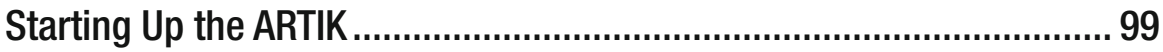

The System Administrator Console ....................................................... 99

U-boot Universal Boot Loader Messages .............................................. 99

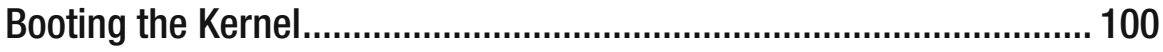

OS Kernel Startup................................................................................... 101

Setting the Boot Mode Switches........................................................ 103

Booting Up Your ARTIK Development System .................................... 104

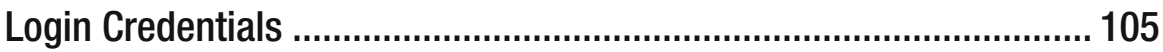

Shutdown Commands ........................................................................... 106

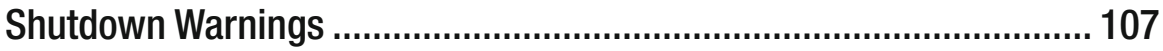

Shutdown Console Logging Messages.............................................. 108

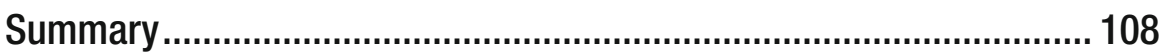

Chapter 9: Network Configuration .......................................... 109

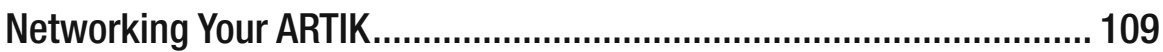

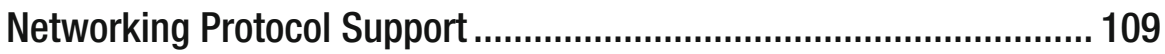

Choosing the Best Networking Strategy ......................................... 110

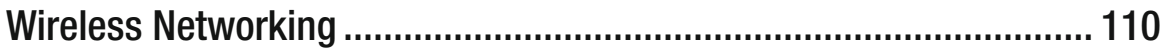

Dynamic Name Auto-discovery Support............................................... 111

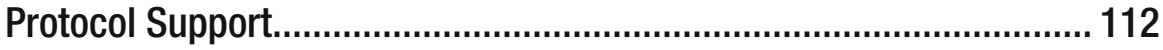


OMA Lightweight M2M Protocol (LW M2M)

Constrained Application Protocol (CoAP)

Message Queue Telemetry Transport Protocol (MQTT)........................ 113

6LoWPAN Protocol................................................................................ 114

Using ZigBee and Thread Protocols.................................................. 114

OpenHAB Support in ARTIK 10 Modules ............................................ 115

OpenStack (Swift) Framework .............................................................. 116

Configuring Your Ethernet Connection ................................................. 117

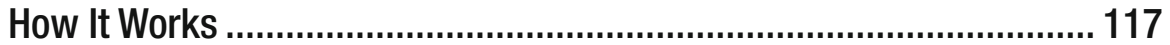

IPv4 Addressing Notation ...................................................................... 118

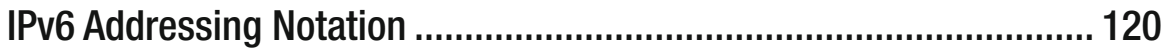

Port Numbers .................................................................................... 121

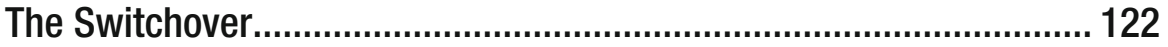

IP Address Configuration in Your ARTIK .............................................. 122

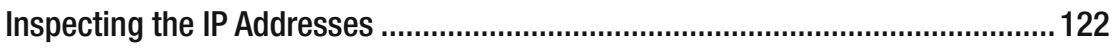

Setting a Temporary IP Address on the Ethernet Interface ................................. 123

Setting a Default Persistent Static IP Address......................................................123

Configuring Your ARTIK for IPv6 Operation ........................................................125

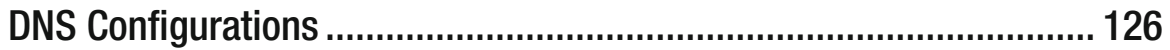

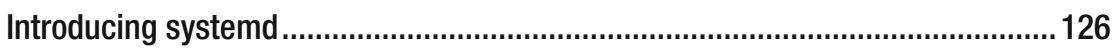

The Impact of systemd on DNS Configuration...................................................126

Statically Configuring Your DNS Servers ..................................................... 127

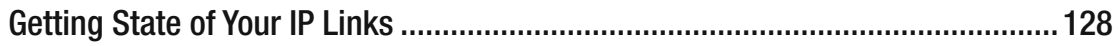

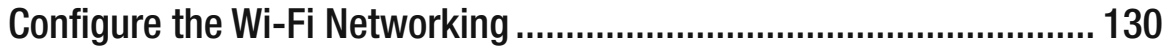

Setting Up Wi-Fi Communications .................................................................130

Another Way to Configure Your Wi-Fi.......................................................... 133 
Troubleshooting FAQ 135

Advanced Wi-Fi Configuration ....................................................................136

Automatically Reconnect Your Wi-Fi after Each Reboot ........................................136

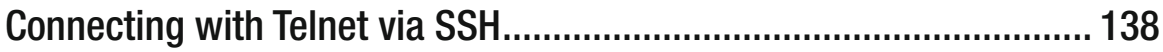

Configuring Your Bluetooth Wireless Interface ................................... 139

Setting Up Bluetooth for an ARTIK 5 or 10 ...................................................... 139

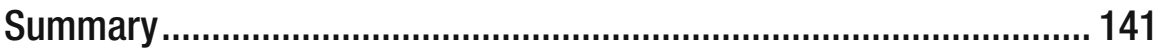

Chapter 10: Configuring and Upgrading ................................. 143

Updating Your Operating System.......................................................... 143

Writing Downloaded Images to an SD Card ....................................... 143

Writing Micro SD Card Images on Windows ......................................................143

Writing Micro SD Card Images on Linux ............................................................. 144

Writing Micro SD Card Images on Mac OS X ....................................................146

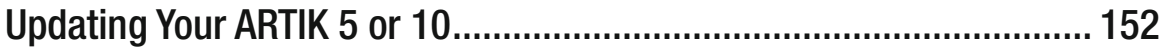

Known Firmware Versions............................................................ 155

Installing Software on Your ARTIK ...................................................... 156

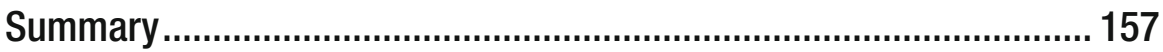

Chapter 11: Programming Your ARTIK................................... 159

Everything Is the Same but Different .............................................. 159

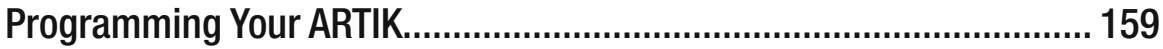

Setting Up Your Software Development Environment .......................... 160

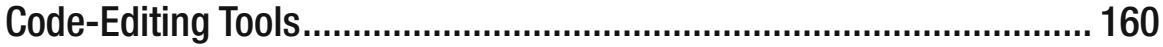

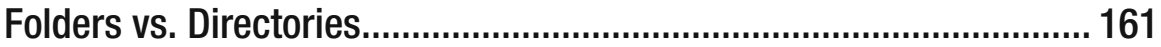

File-System Path: Folder Separator Characters .................................. 161

Spaces in File Names and Paths...................................................... 162

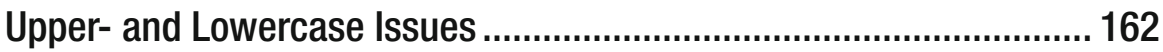


Of Camels and Underscores ........................................................... 164

Let the Environment Do the Heavy Lifting .......................................... 164

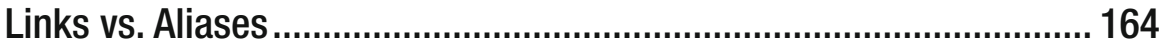

Mac OS Resource Forks .............................................................. 165

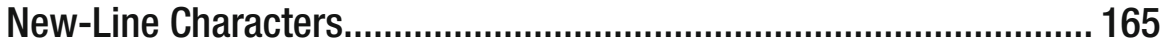

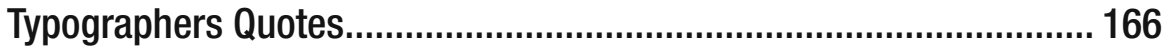

Being in Two Places at Once ........................................................... 166

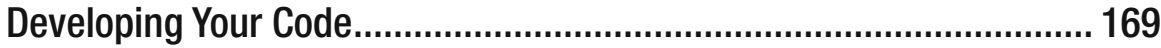

What Is Cross-Compiling? .............................................................. 169

Building Code for the Correct Target CPU ....................................................... 170

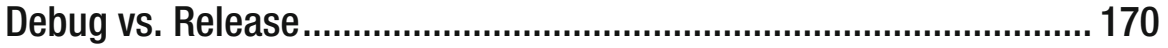

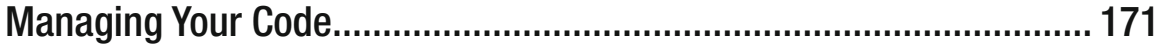

Why Do You Need Java? ................................................................ 172

Checking the Java Version on Windows ............................................................172

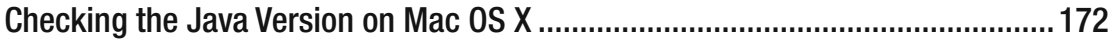

Checking the Java Version on Linux ...............................................................173

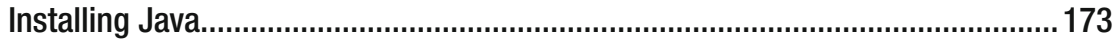

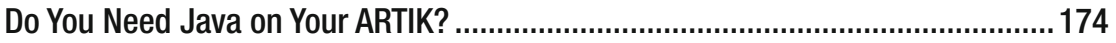

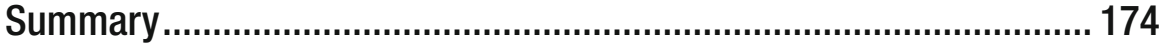

Chapter 12: Using Eclipse IDE ............................................ 175

Installing, Configuring, and Using Eclipse IDE ..................................... 175

Before You Install Eclipse IDE.............................................................. 175

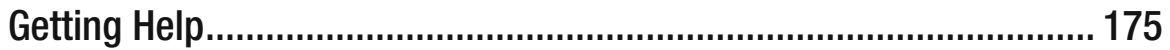

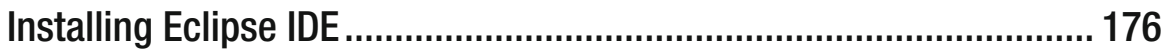

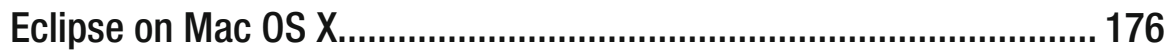

Workspace Preferences ................................................................... 178

Adding New Tools to Your Eclipse IDE .............................................. 179 
What Is a Toolchain? .......................................................................... 180

Installing Support for ARTIK Development.......................................... 180

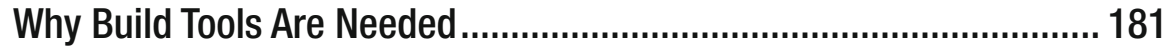

Installing the GNU ARM Eclipse Plugin on Mac OS X.......................... 181

Installing an ARM Toolchain on Mac OS ............................................. 182

Configuring Your IDE for Remote Exploring ....................................... 185

Setting Up a Default Toolchain ............................................................. 188

Semi-hosting Stubs............................................................................... 189

Support for the MIPS Architecture....................................................... 190

Support for Eclipse Smart Home ......................................................... 190

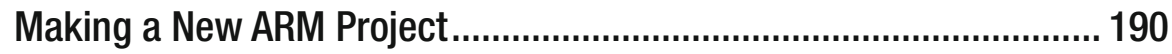

Deploy the Binary to Your ARTIK............................................................ 194

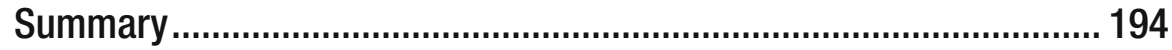

Chapter 13: Using Arduino IDE ............................................. 195

Installing, Configuring, and Using Arduino IDE ................................. 195

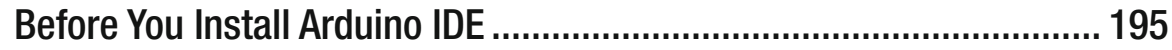

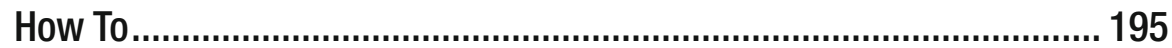

Recommended Settings for Your Arduino IDE ................................... 197

Installing and Configuring libArduino ................................................. 199

Configuring Your ARTIK for Uploads (Board Setup)............................. 201

Uploading a Sketch to Your ARTIK with Arduino IDE ................................202

Network Upload Method ................................................................................. 202

Serial Upload Method .................................................................................... 203

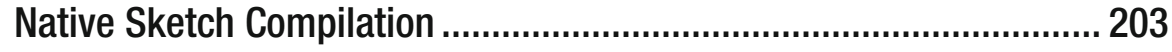

Recommended Update Cycle ........................................................... 204

Developing with libArduino SDK..................................................... 204 
Arduino Pins: Type 1 Developer Reference Board ................................. 204

Arduino Pins: Type 5 and Type 10 Developer Reference Boards.......... 206

System Commands ................................................................... 208

Detecting the Board Version............................................................. 208

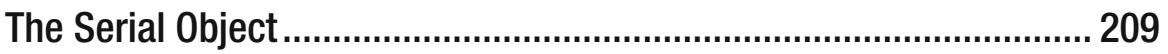

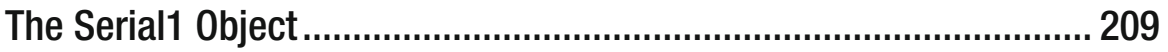

The DebugSerial Object.................................................................. 210

Pin Modes........................................................................ 210

Reading Digital Input Pin Values....................................................... 211

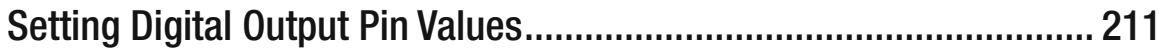

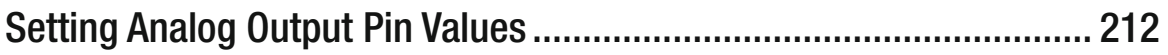

Reading the Analog Inputs ....................................................... 213

Serial Peripheral Interface (SPI) ......................................................... 214

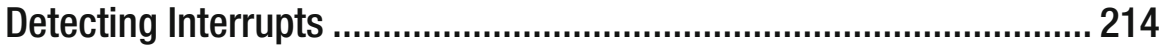

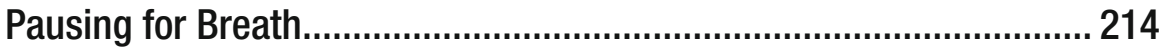

Powersaving Mode ................................................................... 215

Compiling and Running Sketches Natively........................................215

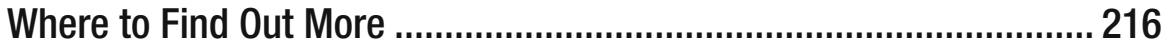

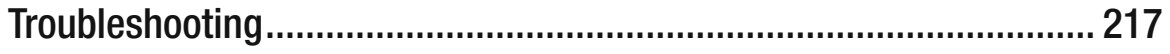

Managing the Type 5 vs. Type 10 Pin-Number Differences ...............................217

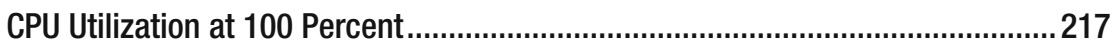

Digital Read Only Ever Reports a 1 Value ............................................................217

Porting Projects from Other Architectures........................................................218

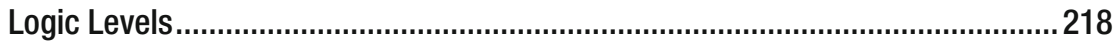

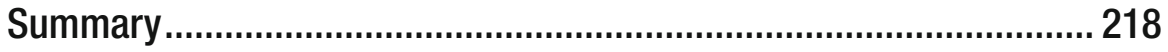


Chapter 14: Using the Command Line 219

Command-Line ARM Toolchains........................................................ 219

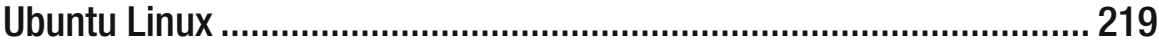

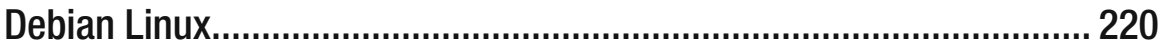

Mac OS X

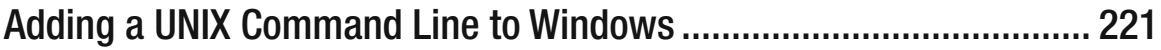

UNIX I/0 Streams and Redirection............................................................ 221

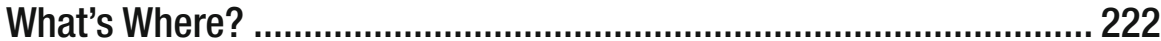

File System Mapped Properties Inside the ARTIK.................................. 223

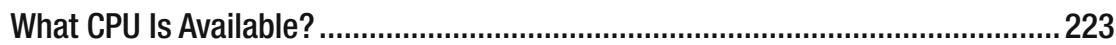

Detecting Current Processor Speed ................................................................224

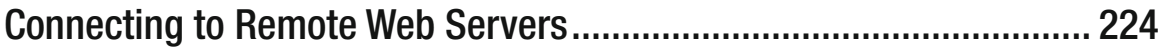

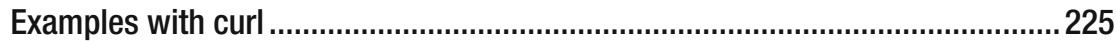

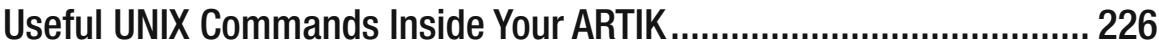

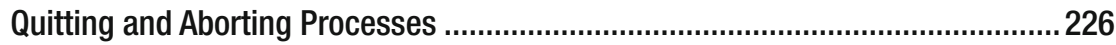

Inhibiting the Debugging Messages ...................................................................227

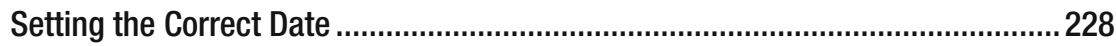

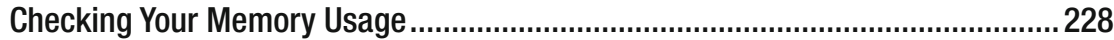

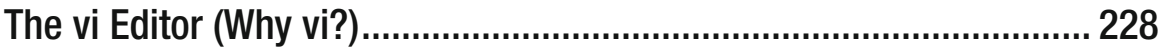

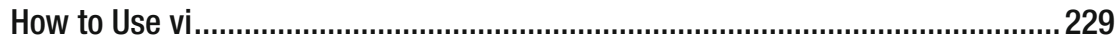

Open a File for Editing or Create a New One ......................................................229

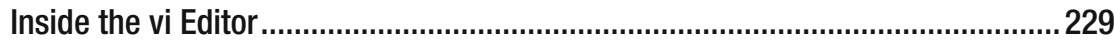

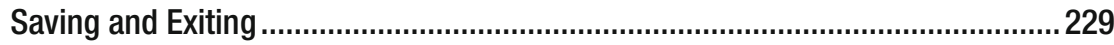

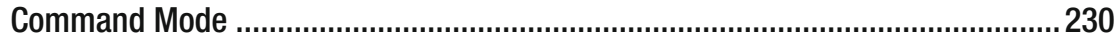

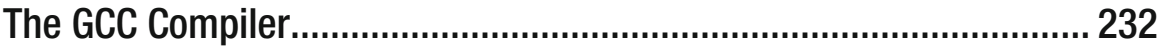

Language Support ................................................................................. 233

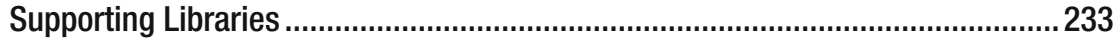

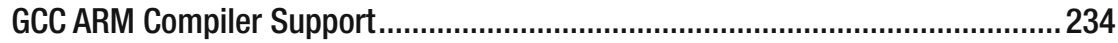




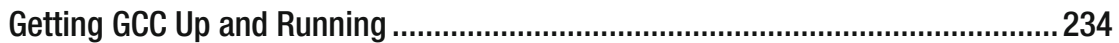

Writing a Simple Program (Hello World) ......................................................2235

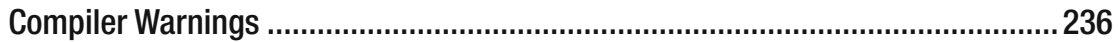

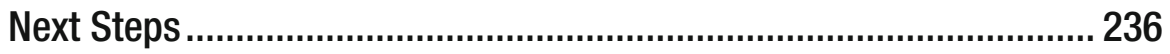

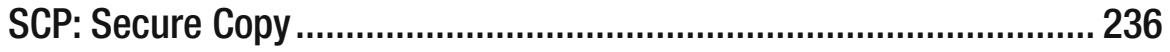

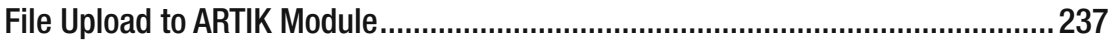

File Download from ARTIK Module ...................................................................237

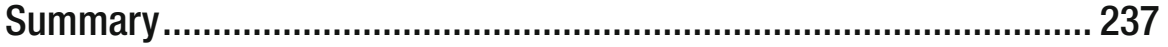

Chapter 15: Programming in C Language.................................. 239

Programming Your ARTIK Natively in C................................................ 239

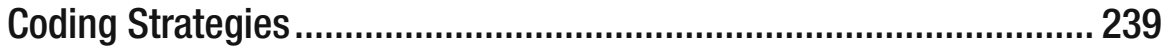

Creating a Simple Application ...................................................... 240

Looking Deeper Inside Your ARTIK...................................................... 241

About the /sys Virtual File System....................................................... 242

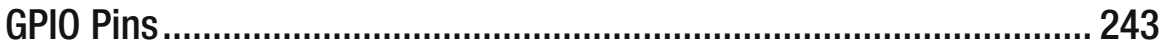

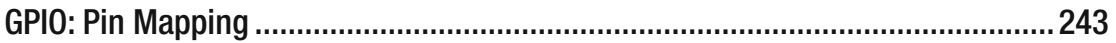

GPIO: Pin Export to the User Domain ............................................................ 245

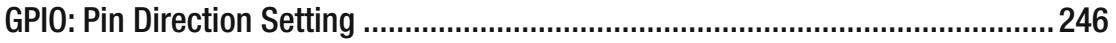

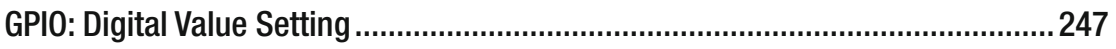

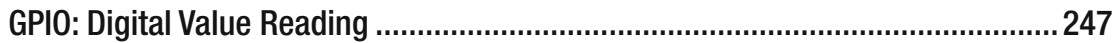

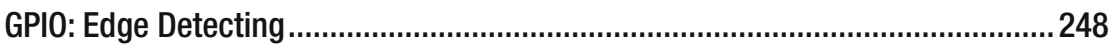

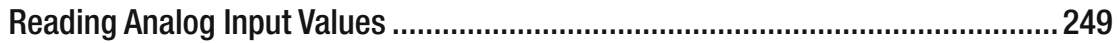

Analog Read Differences Between ARTIK 5 and 10 .........................................250

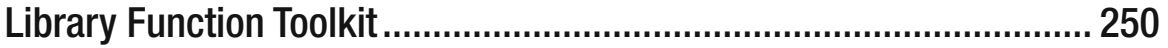

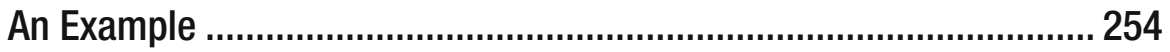

Accessing Remote Systems with libCurl.......................................... 254

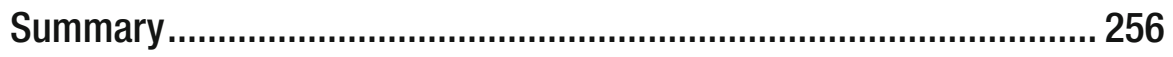


Chapter 16: Programming with Node.js

Developing with Node.js 257

The Architectural Design 258

Compiled Binary Code 259

Checking the Version of Your Node.js Installation .............................. 259

Extending Node.js................................................................................ 260

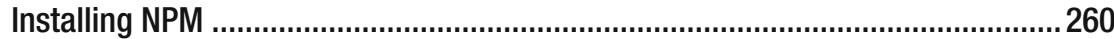

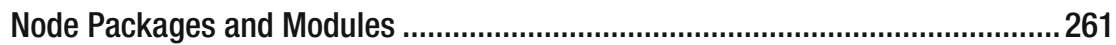

Installing the WebSocket Module .....................................................................262

Let's Write Some Node.js Code ....................................................... 262

Reading a Pin Voltage with Node.js.................................................. 262

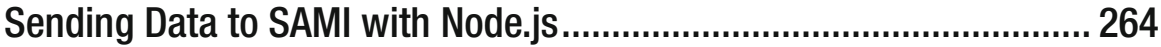

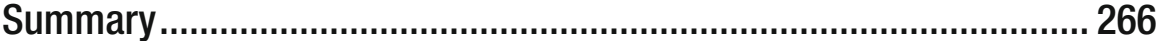

Chapter 17: Programming with Python ................................... 267

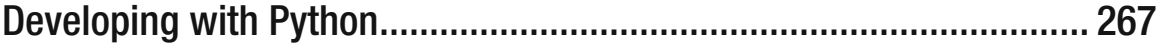

Checking Your Python Interpreter....................................................... 268

Installing the Python Package Manager ............................................. 268

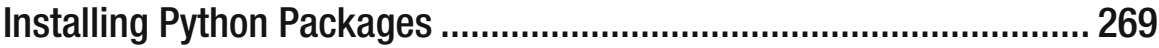

Run a Simple Python Test................................................................ 269

Reading a Pin Voltage with Python................................................... 270

Summary.............................................................................................. 271

Chapter 18: Integrating with SAMI........................................ 273

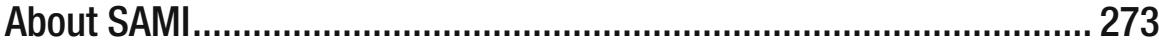

What Is SAMI? ...................................................................................... 274

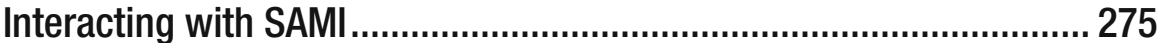

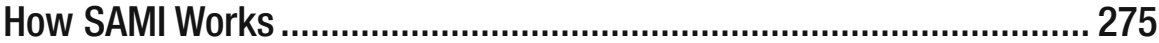


SAMI Developer Documentation....................................................... 276

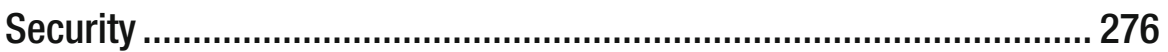

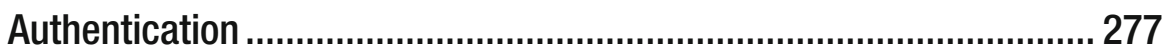

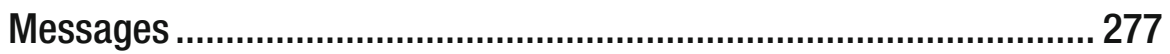

User

User ID

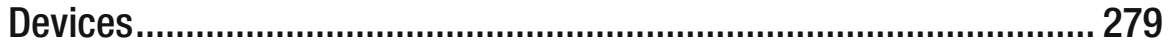

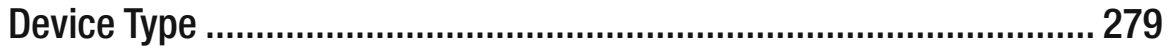

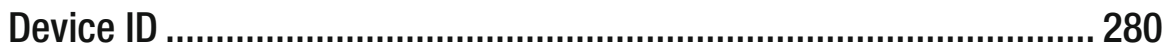

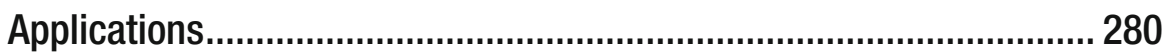

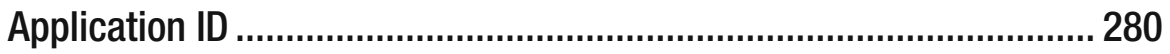

OAuth2 Access Tokens ............................................................ 280

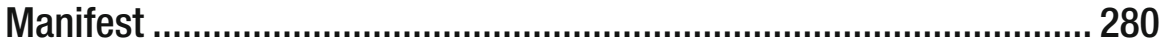

Raw Data

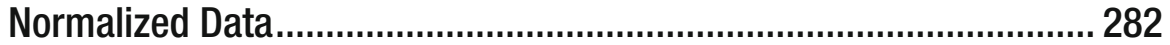

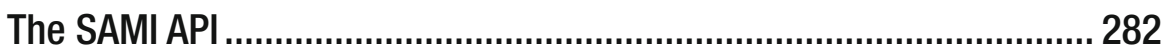

Developer SDK Libraries............................................................. 283

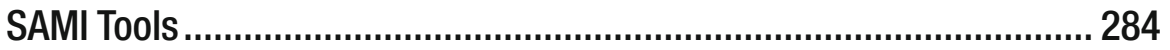

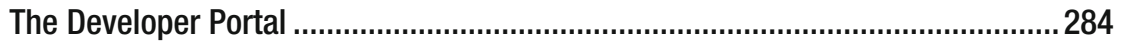

The User Portal .................................................................................. 285

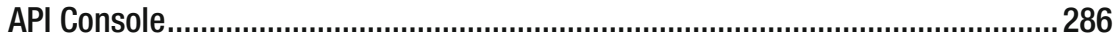

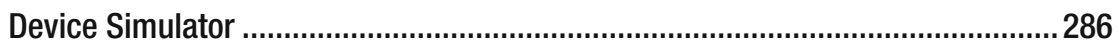

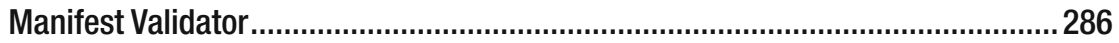

User Portal: Managing Devices ....................................................... 286

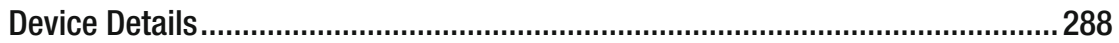




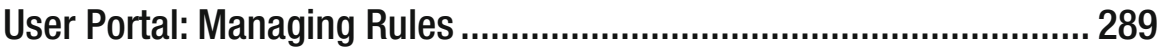

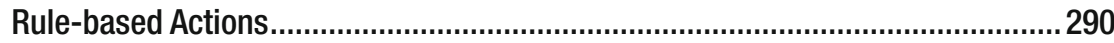

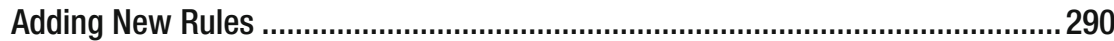

User Portal: Displaying Charts............................................................. 292

User Portal: Viewing Data Logs .......................................................... 293

User Portal: Exporting Data ................................................................ 294

Developer Portal: Managing Device Types.......................................... 294

Developer Portal: Managing Applications............................................ 295

Connecting to SAMI from Your Applications ....................................... 295

Acquiring an Access Token for Your Application.................................. 296

Getting Data from SAMI for Your Application...................................... 296

Sending Data to SAMI from Your Device ............................................. 297

Try Out More Examples ................................................................ 298

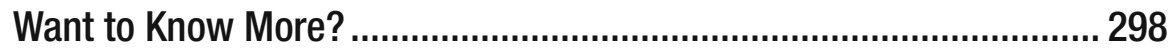

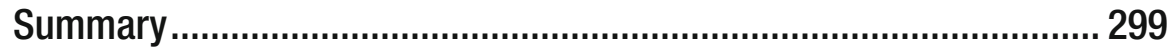

Chapter 19: Integrating with Tembo0 ................................... 301

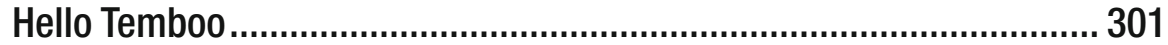

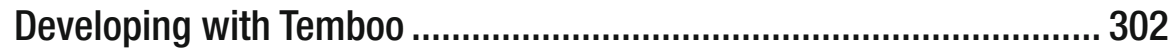

Registering Your Temboo Account ................................................... 302

Your Temboo Account Dashboard ......................................................... 303

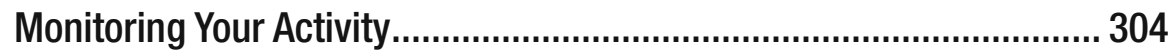

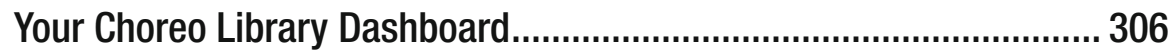

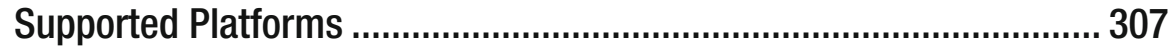

Supported Connectivity ..................................................................... 308 


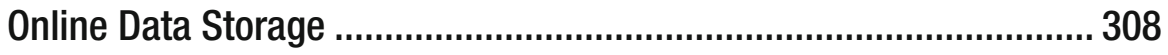

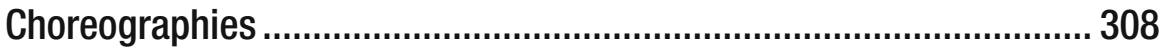

Condition Handling ....................................................................... 310

Remote Storage in Profiles............................................................... 311

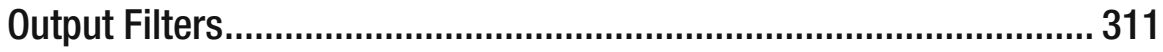

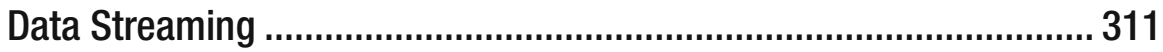

Machine-to-Machine (M2M) with Temboo.......................................... 312

Temb0o and ARTIK..................................................................... 312

Temb0o and ARTIK 5

Getting Ready to Tango with Temboo ….............................................. 313

An Example of Code Generated by Temboo ……………...................... 315

Shared Login Credentials ............................................................... 320

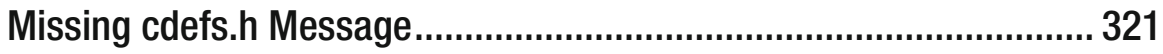

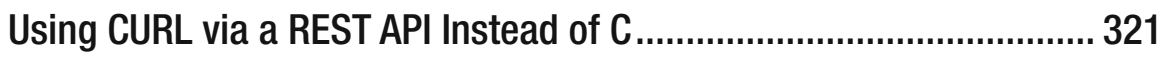

Using Temboo with Node.js ............................................................. 322

Sample Code to Experiment With ....................................................... 322

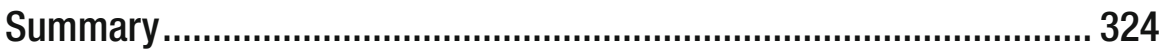

Chapter 20: Debugging Your Application.................................... 325

Debugging Your App ................................................................... 325

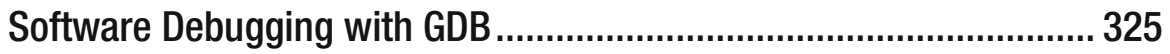

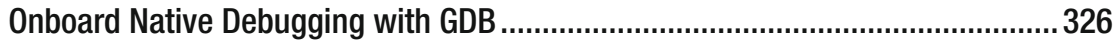

Remote Debugging with GDB ................................................................... 327

IDE Support for Debugging............................................................. 328

Emulating Your Hardware with QEMU ............................................. 329

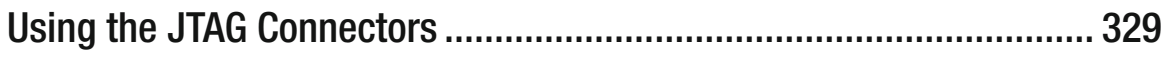


Hardware Debugging with SEGGER J-Link....................................... 329

Hardware Debugging with OpenOCD............................................... 331

Cleaning Up after Debugging .......................................................... 331

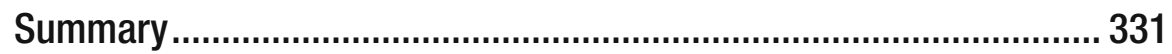

Chapter 21: Deploying Your Application ................................ 333

Getting Ready ............................................................................... 333

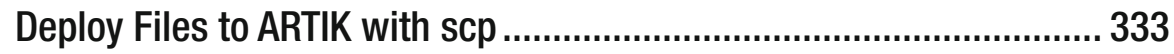

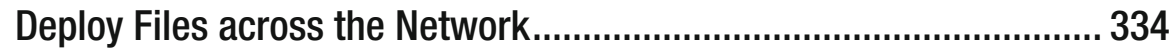

Deploy Files to Your ARTIK with a Micro SD Card ................................ 334

Deploy Files to Your ARTIK with a USB Flash Drive ............................. 337

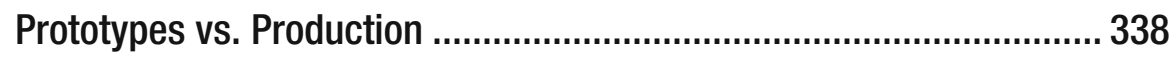

Integrating the ARTIK into your Products ......................................... 338

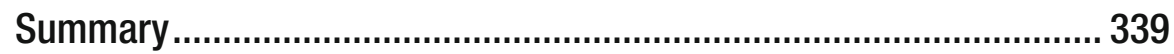

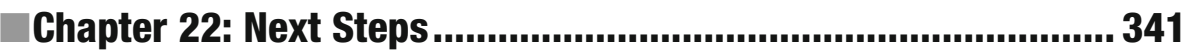

What Do You Want to Make?............................................................ 341

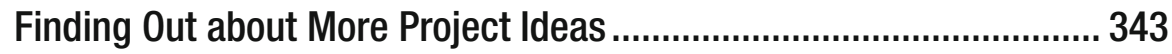

Becoming a Partner Organization...................................................... 344

Going Deeper into ARTIK Development............................................. 344

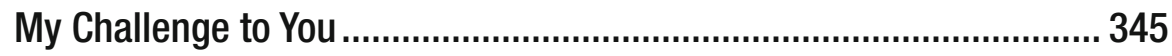

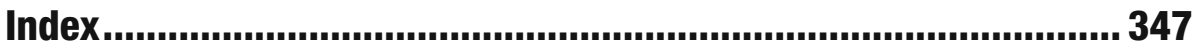





\section{About the Author}

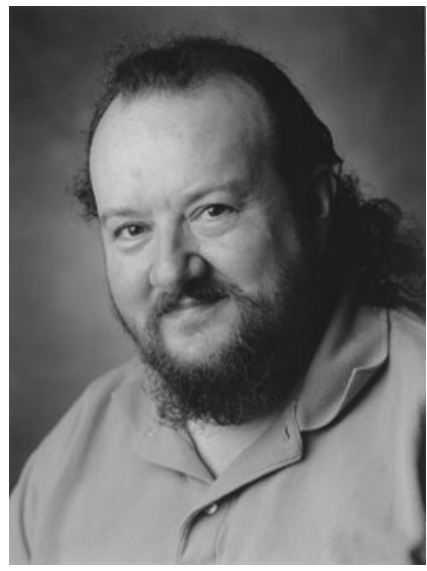

Cliff Wootton is a Royal Television Society (2002) Innovation award-winning former interactive TV systems architect at the BBC, specializing in content management systems and digital video. He was a guest speaker on pre-processing for video compression at the Apple WWDC 2007 developer conference and presented a technical paper on interactive TV systems at the NAB 2003 conference. He has taught IoT, real-world computing with Arduino, multimedia, video compression, metadata, and how to build multimedia art installations at the master's level at the University of the Arts, London. Cliff now concentrates on research and development projects, building digital media tools for creating audiovisual content, multimedia, electronic book publishing, writing, teaching, and playing the bass guitar. 



\section{Acknowledgments}

Huge thanks are due to these people who created resources online and contributed ideas and practical help while I was writing this book. Their assistance was truly invaluable and is gratefully acknowledged.

- $\quad$ Steve Weiss, Melissa Maldonado, Jeff Pepper, and Jonathan

Gennick at Apress-it has been great to work with you guys again

- Glenn Cameron at Samsung, who kindly provided prototype hardware and technical resources

- Curtis Sasaki for generously contributing the foreword

- $\quad$ Fred Patton at Samsung for his excellent photographic contributions and technical support

- Martin Kronberg for putting some helpful projects online in the Hackster.io blog

- Simon Tatham, who wrote and maintains the PutTTY application

- $\quad$ The minicom project team

- The Temboo team for their generosity in sharing insights into deeply technical interface code through their code-publishing mechanisms

- Kevin Sharp for his helpful contributions to the ARTIK blog

- All the artik.io forum posters whose questions inspired me to cover interesting topics

- Gaynor Bromley at Panasonic Electric Works UK Ltd for the AXT connector images

- Joe Geoghegan and Bhavin Naik at Mentor Graphics for technical white papers about Nucleus OS

- Paul Stoffregen for developing the Bridge. $h$ library used in the Arduino IDE

- Tevon Jordaan for introducing me to Scrivener (an extraordinarily good book-writing tool).

- The Scrivener application developer team (see http://www.literatureandlatte.com) 



\section{Trademarks}

$\mathrm{ARM}^{\bullet}$ is a registered trademark owned by ARM Ltd.

Mali $^{\text {mt }}$ is a trademark owned by ARM Ltd.

MIPS $^{\circledR}$ microAptiv $^{\text {mat }}$ is a trademark owned by Mentor Graphics.

Linux ${ }^{\odot}$ is the registered trademark of Linus Torvalds in the United States and other countries.

$\mathrm{Java}^{\circ}$ is a registered trademark of Oracle and/or its affiliates.

All other trademarks are the property of their respective owners. 



\section{Foreword}

If you follow the tech media at all, you will no doubt have seen plenty of coverage of-and excitement for-the Internet of Things (IoT). The hype surrounding IoT has been palpable. But now we appear to have reached an inflection point, where the hype is becoming reality. IoT is transforming the way people live their lives, with the power to automate, connect, and inform us like never before.

The technology to support IoT is evolving rapidly, with the availability of new, highly-integrated "systems on modules" (SOMs), as well as optimized wireless support and diverse sensor technologies. The most important change however, may be the move back to hardware. Over the past few years, most innovation has been taking place in the cloud and in system software. But with IoT, we are seeing renewed energy and commitment to hardware development. The arrival of low-cost development platforms, such as Arduino and Raspberry Pi, has helped the burgeoning maker community to really take off.

The Samsung ARTIK IoT platform takes this evolution to the next phase with its System on Module (SOM) concept. Designed for both individual developers and those who need it for large-scale production purposes, it features not only the application processor, but also DRAM, flash memory, radios, and advanced security options.

The idea behind ARTIK is simple: reduce the complexity of designing your IoT hardware. Previously, designers often had to do their job twice-once to get a working prototype, and then a redesign for the final production board. With ARTIK, you have the advantage of using the same module in your prototype that you will use in the final production hardware.

Beyond the hardware, the ARTIK platform also delivers a full stack with the latest drivers and tools, as well as a cloud service. And because ARTIK is an open platform, the choice of software is up to you.

As you can imagine, ARTIK is designed to support a broad range of uses, from wearables and products for the smart home to powerful hubs capable of local processing and analytics.

All of us at Samsung are excited to see the creativity that you will bring in leveraging the ARTIK platform, and how your work can change the world around us in positive ways. Of course, having books and documentation to help you get started is always welcome.

We believe that this Beginning Samsung ARTIK book and the companion ARTIK Reference Guide will provide plenty of inspiration to help you achieve your vision with the ARTIK platform.

-Curtis Sasaki

Samsung ARTIK project leader, Spring 2016 



\section{Introduction}

This introductory book will help you start your journey toward becoming an expert ARTIK developer as you develop a new and profitable enterprise.

This book will show you how to set up your own ARTIK development system and get your ARTIK module up and running. Then you can develop your own applications for it. By the end of the book you should have a working system and be able to create and deploy simple applications to your ARTIK module. Then it will be up to you to create something extraordinary. The chapters are arranged in a logical sequence starting with some background information, then how to set up your system, before describing basic programming techniques. External systems such as Temboo and SAMI are covered before rounding things off with debugging and deployment guidelines.

I composed this as I got to know the ARTIK after Glenn Cameron from Samsung kindly sent one to me to work on. As I found out new and interesting things about it, I wrote about what I learned straightaway while the knowledge was still fresh. I tried to maintain the perspective of a new user encountering ARTIK for the first time to avoid making assumptions about what the reader might already know. Some readers will already know about the topics I cover. Even so, the additional background information I provide here will be helpful even to more experienced engineers, designers, makers, and developers.

It is still an early stage in the ARTIK product lifecycle, and many features are still being developed to their full potential. There are topics like video and audio that merit complete books about just that aspect of ARTIK development. Those will come later. For now, it is enough to understand the basics of how ARTIK works so as to have solid foundational knowledge on which to later build the more advanced topics. Some of those topics are mentioned briefly here, but there is not enough space to cover everything in great depth without creating a huge book. For now, let's work on this in more easily digestible stages and get the ARTIK modules up and running first.

The companion ARTIK Reference Guide will build on what you learn in this book. It will concentrate on ARTIK internals and provide details for more advanced programming so that you can create more complex applications. 